\title{
Satisfação de usuários de planos odontológicos: um estudo de caso em uma operadora de autogestão
}

\author{
Satisfaction of dental plan users: a case study in a self-management health \\ insurance company
}

\author{
Alyne Silveira Pereira \\ Daniela Garbin Neumann** \\ João Carlos Caetano*** \\ Mirelle Finkler ${ }^{* * *}$ \\ Denise Stuart Mussi*****
}

\section{Resumo}

Introdução: o setor de saúde suplementar iniciou suas atividades no final da década de 1980 e, atualmente, seus usuários somam 51 milhões no segmento médico e mais de 21 milhões na área odontológica. Devido ao crescimento da odontologia na saúde suplementar, torna-se necessário conhecer os fatores que interferem na qualidade dos serviços oferecidos para, assim, implantar práticas que os aperfeiçoem. Objetivo: este estudo de caso de abordagem quantitativa teve como objetivo identificar o grau de satisfação de usuários vinculados a uma operadora de autogestão frente à assistência odontológica recebida. Sujeitos e método: os participantes da pesquisa foram usuários com mais de 18 anos que compareceram à sede da operadora durante o período de coleta de dados e aceitaram participar do estudo ( $n=175)$, respondendo a um questionário. Resultados: os usuários consideram seu plano de saúde bom ou excelente $(80,6 \%)$. A maioria declarou satisfação com a assistência odontológica recebida em relação ao custo-benefício $(64,5 \%)$, à infraestrutura $(86,8 \%)$, à qualidade do tratamento $(84,5 \%)$ e aos prazos de atendimento $(78,8 \%)$, sendo que $82,9 \%$ recomendariam a operadora. Conclusão: há um alto grau de satisfação por parte dos usuários do plano vinculado à operadora de autogestão, ainda que, de acordo com a literatura, os profissionais não têm demonstrado a mesma satisfação quanto à sua atuação no setor de saúde suplementar.

Palavras-chave: Avaliação de serviços de saúde. Saúde suplementar. Seguro odontológico.

\section{Introdução}

A criação do Sistema Único de Saúde (SUS), com a promulgação da Constituição federal em 1988, institucionalizou o direito à saúde a todos os cidadãos do país. Ao se afirmar a saúde como dever do Estado, surge a oportunidade de efetivação de um sistema integrado, universal e gratuito que cuida da prevenção, promoção, cura e reabilitação da população brasileira, com vistas ao fortalecimento da cidadania ${ }^{1}$, mas os investimentos setoriais não acompanharam o aumento da cobertura de prestação de serviços. Assim, houve um crescimento de modalidades de seguros e planos de saúde ${ }^{2}$, o que acabou por caracterizar o sistema que deveria ser único como plural e segmentado, formado por três subsistemas: o SUS, o Sistema de Atenção Médica Supletiva ou de Saúde Suplementar (SS) e o Sistema de Desembolso Direto. O primeiro é um sistema público, os dois últimos, sistemas privados ${ }^{3}$.

Durante a década de 1990, observou-se que a SS teve um crescimento desordenado e desregulado, o que levou à aprovação da Lei no 9.656/1998, que dispõe sobre os planos e seguros privados de assistência à saúde, e, posteriormente, da Lei no 9.961/2000, que criou a Agência Nacional de Saúde Suplemen-

http://dx.doi.org/10.5335/rfo.v21i1.5526

Cirurgiã-dentista.

Doutora em Odontologia em Saúde Coletiva.

Doutor em Saúde Pública, Departamento de Saúde Pública, Programa de Pós-Graduação em Odontologia, Universidade Federal de Santa Catarina, Florianópolis, Santa Catarina, Brasil.

Doutora em Odontologia em Saúde Coletiva, Departamento de Odontologia, Programa de Pós-Graduação em Odontologia, Universidade Federal de Santa Catarina, Florianópolis, Santa Catarina, Brasil.

Mestre em Odontologia em Saúde Coletiva. 
tar (ANS), autarquia sob regime especial vinculada ao Ministério da Saúde ${ }^{1,4}$. A partir de então, o crescimento da SS foi ainda mais evidente, havendo hoje quase 51 milhões de usuários de planos de assistência médica e 21 milhões de usuários de planos exclusivamente odontológicos ${ }^{5}$.

Embora se trate de um mercado regulado pela ANS, a conformação da SS também está sujeita à microrregulação, que representa as formas de regulação que se estabelecem entre operadoras, prestadores e usuários, sem uma clara intermediação do Estado ${ }^{6}$. A regulação representada pela ANS e a microrregulação exercida pelas operadoras delineiam os limites contratuais e modulam a produção do cuidado ${ }^{7}$.

Os espaços microrregulatórios na SS e, mais especificamente, na sua segmentação odontológica já foram objeto de estudo de alguns pesquisadores, tanto sob o ponto de vista das operadoras ${ }^{8,9}$ como dos prestadores ${ }^{10,11}$ e dos usuários ${ }^{8}$. Esses estudos revelaram resultados contraditórios relacionados à odontologia na SS, como percepções positivas de usuários quanto à assistência recebida ${ }^{7}$ contrapondo-se à insatisfação de prestadores frente à perda de autonomia em seu processo de trabalho, à burocracia e à baixa remuneração $0^{10,11}$. Ainda, constataram uma grande preocupação com redução de custos por parte das operadoras ${ }^{8,9}$ em detrimento de uma gestão que deveria priorizar um modelo de atenção à "saúde bucal" voltado à integralidade. No entanto, não esgotaram a necessidade de se conhecer as peculiaridades da segmentação odontológica da SS nos espaços microrregulatórios sob o olhar dos diferentes participantes.

Uma das formas de desvendar aspectos relacionados à qualidade da atenção na área da saúde é por meio da percepção, avaliação e satisfação do principal interessado pelo serviço prestado - o usuário ${ }^{12}$. No âmbito institucional da SS, pesquisas com usuários sobre o preço dos planos contratados, estrutura, prazos dos serviços, comunicação e expectativas são realizadas como ferramenta para avaliação e qualificação das operadoras, sendo inclusive parte componente do Índice de Desempenho da Saúde Suplementar (IDSS), o qual serve de ferramenta para avaliação das operadoras em diferentes aspectos e é divulgado periodicamente pela $\mathrm{ANS}^{13,14}$.

Apesar da existência de alguns estudos abordando a satisfação de usuários, ainda são poucos os específicos sobre a assistência odontológica. Este estudo buscou identificar a satisfação de usuários vinculados a uma operadora de autogestão frente à assistência odontológica recebida, a fim de compreender melhor os aspectos microrregulatórios da odontologia da SS.

\section{Sujeitos e método}

Este estudo de caso com abordagem quantitativa foi aprovado pelo Comitê de Ética em Pesquisa com Seres Humanos da Universidade Federal de Santa Catarina (parecer no 831.362). Os participantes da pesquisa eram usuários de planos de assistência médica com cobertura odontológica, vinculados a uma operadora de autogestão com abrangência nacional. A sede considerada para a pesquisa foi a do município de Florianópolis, Santa Catarina, que, em março de 2014, contava com 10.276 usuários cadastrados ${ }^{15}$.

A pesquisa foi realizada por meio da aplicação de um questionário previamente testado, com perguntas fechadas de múltipla escolha, elaborado com base no instrumento utilizado pela ANS em sua Pesquisa de Satisfação dos Beneficiários ${ }^{16}$. As questões foram adaptadas para as especificidades da assistência odontológica, sendo as alternativas construídas com base em respostas em escala do tipo Likert ${ }^{17}$.

A coleta de dados se deu na própria sede da operadora, entre novembro de 2014 e fevereiro de 2015, durante doze horas por semana. Nesse período, cada usuário que compareceu ao local e que se enquadrava nos critérios de inclusão - possuir plano com cobertura odontológica, residir na região metropolitana de Florianópolis e ter pelo menos 18 anos - foi convidado a participar da pesquisa. Os questionários foram entregues pela pesquisadora aos participantes, que o responderam sem qualquer intervenção.

Foram abordados 383 usuários, sendo que 208 não participaram da pesquisa, por não se encaixarem nos critérios de inclusão e/ou pelo não aceite de participação. Assim, 175 usuários responderam ao questionário. Os dados coletados foram tabulados e analisados de forma descritiva, sendo a seguir apresentados e discutidos.

\section{Resultados e discussão}

\section{Perfil dos participantes}

A maior parte da amostra foi composta por mulheres $(64 \%, 112)$ na faixa etária entre 51 e 70 anos $(59,4 \%, 104)$. Quanto à escolaridade, apenas $4,6 \%$ (8) tinham somente o ensino fundamental, enquanto $60 \%$ (105) tinham ensino superior. Em relação à renda, 54,9\% da amostra (96) possuía renda familiar mensal entre três e nove salários mínimos e $41,1 \%$ (72), mais de nove salários mínimos. A Tabela 1 apresenta o detalhamento do perfil da amostra. 
Tabela 1 - Perfil dos participantes segundo faixa etária, sexo, escolaridade e renda, Florianópolis, 2015

\begin{tabular}{l|r|r}
\cline { 2 - 3 } & \multicolumn{1}{|c|}{$\mathrm{N}$} & \multicolumn{1}{c}{$\%$} \\
\hline Faixa etária & 17 & 9,7 \\
18 a 30 anos & 34 & 19,4 \\
31 a 50 anos & 104 & 59,4 \\
51 a 70 anos & 20 & 11,4 \\
71 anos ou mais & & \\
Sexo & 112 & 64,0 \\
Feminino & 63 & 36,0 \\
Masculino & & \\
Escolaridade & 62 & 4,6 \\
Ensino fundamental & 105 & 60,0 \\
Ensino médio & & \\
Ensino superior & 7 & 4,0 \\
Renda & 96 & 54,9 \\
Até 3 salários mínimos & 72 & 41,1 \\
Mais de 3 até 9 salários mínimos & & \\
Mais de 9 salários mínimos &
\end{tabular}

\section{Utilização do plano de saúde para assistência odontológica e avaliação geral}

$\mathrm{O}$ atendimento odontológico mais recente da maioria, 79,4\% da amostra (139), foi pelo plano de saúde. Como salienta Neumann ${ }^{9}$, o crescimento na procura por serviços de saúde bucal no âmbito da SS pode ser sustentado pela dificuldade de acesso à assistência odontológica de financiamento público e/ou pelos custos envolvidos no desembolso direto. Pinheiro e Torres ${ }^{18}$ identificaram que, no Brasil, o maior percentual de acesso a serviços odontológicos foi constatado entre mulheres, em pessoas com mais escolaridade, com maior renda familiar per capita, em usuários de plano de saúde e em residentes de área urbana, dados semelhantes à caracterização da amostra deste estudo.

Grande parte dos respondentes $(80,6 \%)$ considerou seu plano de saúde excelente ou bom, conforme ilustra a Figura 1. A avaliação positiva dos participantes também parece se relacionar com a taxa de utilização da assistência odontológica por meio do plano de saúde. Esses resultados concordam com os apresentados por Barros e Bertoldi ${ }^{19}$, que, em uma avaliação em nível nacional sobre acesso e utilização dos serviços odontológicos, encontraram alta proporção do julgamento como "bom ou muito bom", tanto entre os pacientes que pagaram pelo serviço (desembolso direto) quanto entre os que foram atendidos pelo SUS.

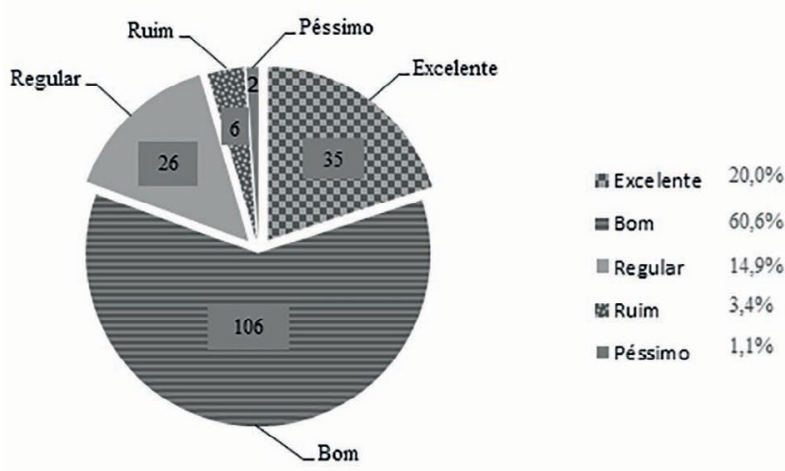
Figura 1 - Avaliação do plano de saúde pelos participantes, Florianó-
polis, 2015

Fonte: dados da pesquisa.

Visto que a pesquisa foi realizada apenas com usuários com cobertura odontológica, chama a atenção que mais de $20 \%$ (36) dos participantes não tenham realizado sua última consulta odontológica por meio do plano de saúde. Desses, dois participantes responderam ter recebido tratamento de cirurgião-dentista membro da família, quatro por meio do SUS e trinta optaram por tratamento no setor privado. Além das questões de preferência, falta de cobertura ou vínculo familiar, para justificar a procura de profissionais não credenciados à operadora, a inexistência de um prestador responsável pelo acompanhamento dos usuários acarreta em uma descontinuidade da atenção e, com isso, na fragmentação do cuidado ${ }^{7}$.

Sobre a recomendação do plano de saúde para familiares, amigos ou conhecidos, na pesquisa realizada pela $\mathrm{ANS}^{16}, 67,8 \%$ dos participantes afirmaram que recomendariam o plano, $32,3 \%$ não o recomendariam e apenas $1,5 \%$ não soube responder. Neste estudo, no que concerne ao plano de saúde odontológico, 82,9\% (145) dos participantes responderam que o recomendariam, $8,6 \%$ (15) não o recomendariam e 8,6\% (15) não souberam responder.

Salienta-se que essa operadora, por ser da modalidade de autogestão, não permite a inscrição de usuários por indicação, apenas por meio de pessoas jurídicas, as quais, mediante convênio por adesão, passam a ter acesso aos planos de saúde administrados pela operadora, podendo ou não patrocinar em parte o pagamento desses planos. No entanto, essa questão foi mantida apenas como parâmetro de satisfação e para comparação à pesquisa nacional.

\section{Satisfação}

Levando-se em consideração a assistência odontológica, os participantes foram questionados quanto ao preço do plano e aos serviços oferecidos, às opções e à infraestrutura de clínicas e consultórios credenciados, à qualidade dos serviços prestados e a respeito dos prazos para a realização de consultas e exames. Considerando uma escala de "muito sa- 
tisfeito" a "muito insatisfeito", os usuários puderam apontar o nível de satisfação para cada característica relacionada, cujos resultados estão ilustrados na Figura 2.

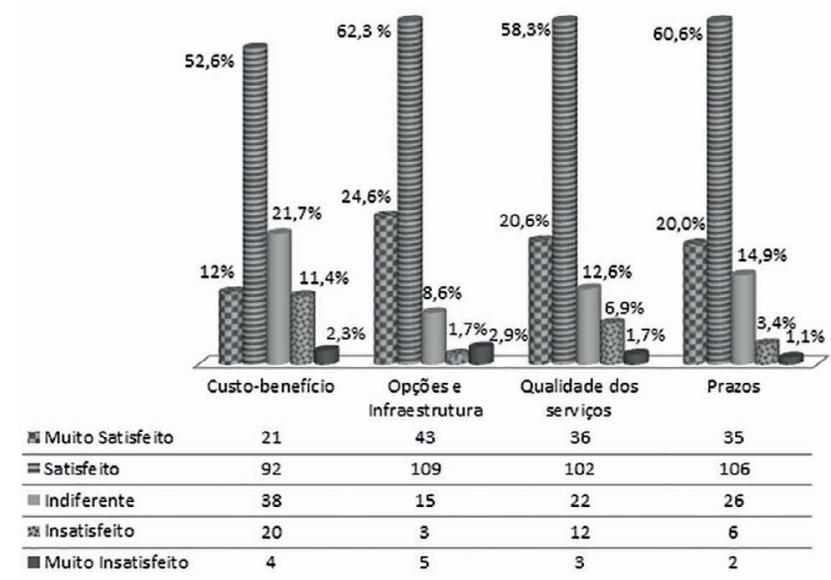

Figura 2 - Satisfação dos usuários quanto a diferentes características da assistência odontológica, Florianópolis, 2015

Fonte: dados da pesquisa.

Para facilitar a comparação com os resultados da pesquisa nacional, optou-se por agregar as respostas "muito satisfeito" e "satisfeito" e considerá-las como satisfeito, bem como as alternativas "insatisfeito" e "muito insatisfeito", que foram consideradas como insatisfeito. Assim, a maioria dos participantes declarou estar satisfeita quanto às características apontadas: em relação ao custo benefício $(64,6 \%)$, às opções e à infraestrutura (86,9\%), à qualidade dos serviços $(78,9 \%)$ e aos prazos $(80,6 \%)$. Levando-se em consideração a mesma soma de respostas, a pesquisa nacional de satisfação realizada pela $\mathrm{ANS}^{16}$ também apontou índices consideravelmente altos, respectivamente: $41 \%, 48 \%, 59 \%$ e $46 \%$. Entretanto, há desarmonia ao se comparar os resultados com o IDSS de 2013 da operadora objeto deste estudo ${ }^{14}$, uma vez que a categoria "satisfação do beneficiário" pontuou apenas 0,2580, ou seja, em torno de $25 \%$ da nota máxima.

Vale ressaltar que esse resultado representa a operadora em nível nacional e que a avaliação leva em conta o plano de assistência como um todo, não sendo especificamente relacionado à assistência odontológica. Além disso, essa categoria engloba o índice de reclamações dos beneficiários, a proporção de desistência no primeiro ano e as infrações cometidas pela operadora, parâmetros que não foram avaliados nesta pesquisa.

A pesquisa realizada pelo Conselho Nacional de Secretários de Saúde ${ }^{1}$ evidenciou que a maior parte dos usuários de planos de saúde declarou estar satisfeita com o atendimento recebido, principalmente devido à rapidez do acesso aos serviços e ao bom atendimento, corroborando os achados desta pesquisa. No estudo de Gerschamn et al. ${ }^{20}$, o aspecto que mais contribuiu com a satisfação dos usuários foi a boa estrutura dos hospitais vinculados ao plano. Da mesma forma, neste estudo, o maior julgamento de satisfação foi relativo às opções e à infraestrutura de clínicas e consultórios odontológicos oferecidos pelo plano de saúde. Cartaxo e Santos ${ }^{21}$ também encontraram um alto grau de satisfação dos usuários de plano odontológico. Os autores sugeriram uma provável relação com o componente financeiro e com a certeza de realização do tratamento disponibilizado pelo plano.

A modalidade de autogestão não tem fins econômicos, sendo um segmento não comercial do setor da $\mathrm{SS}^{22}$, que assume o risco da variabilidade dos gastos com o consumo de serviços de saúde. A pesquisa da Unidas Autogestão em Saúde para o segmento de autogestão constatou que os procedimentos mais realizados no âmbito das operadoras pesquisadas foram os relacionados à prevenção de doenças bucais, como profilaxia e exames radiológicos ${ }^{23}$. Assim, pode-se pensar que a ausência de interesse pelo lucro e, ao mesmo tempo, a necessidade de evitar o uso desnecessário dos serviços possibilitariam um modelo de atenção mais voltado à promoção da saúde e à prevenção de doenças, em detrimento de um modelo curativo e que se utilize de tecnologias com maior custo agregado ${ }^{24}$. Essa característica poderia, então, explicar em parte os resultados encontrados, porém não é possível afirmar que esse modelo seja realmente utilizado pela operadora que foi objeto deste estudo.

\section{Conclusão}

Realizar avaliações quanto a diferentes aspectos do sistema de saúde a partir da perspectiva dos usuários contribui para a constante readequação tanto das práticas profissionais quanto das formas de organização dos serviços, visando ao seu aprimoramento e à qualificação da atenção à saúde.

Levando-se em conta a operadora e a região pesquisada, este estudo evidenciou um alto grau de satisfação entre os usuários com relação à assistência odontológica vinculada ao seu plano de saúde. Esse resultado corrobora outras pesquisas sobre satisfação de usuários no âmbito da SS, porém levanta questionamentos sobre quais mecanismos microrregulatórios podem estar envolvidos nas contradições que se estabelecem nos espaços relacionais, uma vez que os profissionais não têm demonstrado a mesma satisfação quanto à sua inserção nesse mercado.

Diante disso, fica clara a importância da realização de novos estudos de satisfação, como forma de comparação temporal e ferramenta de qualificação. No setor odontológico, torna-se relevante o aprofundamento no conhecimento sobre os motivos de satisfação e insatisfação dos usuários de planos de saúde, devido às particularidades do atendimento, tais como o estreito relacionamento paciente-profissional e a possibilidade de fidelização. 
Em razão do delineamento metodológico adotado nesta pesquisa, os resultados encontrados não podem ser generalizados, mas apenas considerados no universo da operadora pesquisada e interpretados nesse contexto. No entanto, esses dados contribuem para maior compreensão da odontologia na SS. Cabe salientar ainda que este estudo veio a complementar a pesquisa desenvolvida por Neumann ${ }^{9}$, destacando a importância de desvendar os espaços relacionais da SS em suas nuances. Aponta-se a necessidade da estruturação das operadoras e o preparo dos prestadores, para além de fornecer serviços, gerir o cuidado qualificado da atenção à saúde dos usuários.

Finalmente, mesmo que se considere a SS uma alternativa para quem ainda não tem acesso aos serviços do sistema público e/ou aos serviços pagos por desembolso direto, há a necessidade de maior regulação e constante aprimoramento do modelo de atenção praticado, enfatizando o direito dos usuários à saúde bucal de qualidade e em consonância com o modelo do Sistema Único de Saúde.

\section{Agradecimento}

Ao $\mathrm{CNPq}$, pelo apoio financeiro para coleta e análise dos dados desta pesquisa, contemplada na Chamada № 10/2012 MCTI/CNPq/MS - SCTIE DECIT - Pesquisa em Saúde Bucal.

\section{Abstract}

Objective: The Supplementary Health sector was formed in the late 80s and its users currently account for 51 million in the medical segment and over 21 million in the dental field. Due to the growth of dentistry in Supplementary Health, it is necessary to know the factors that affect the quality of services offered in order to implement practices that further improve them. Thus, this quantitative approach of the case study aimed to identify the level of satisfaction of users linked to a self-management health insurance company regarding the dental care received. Subjects and method: The research participants were users older than 18 years who attended the company's headquarters during the data collection period and agreed to participate in the study $(n=175)$ by answering a questionnaire. Results: Users consider their health plan good or excellent (80.6\%). The majority claimed to be satisfied with the dental care received regarding cost-effectiveness (64.5\%), infrastructure $(86.8 \%)$, quality of care $(84.5 \%)$, and fulfillment of deadlines $(78.8 \%)$, and $82.9 \%$ of them would recommend the provider. Conclusion: There is a high degree of satisfaction of plan users linked to a self-management health insurance provider, even though, according to the literature, professionals have not shown the same satisfaction about their activities in the Supplementary Health sector.

Keywords: Dental Insurance. Healthcare Assessment. Supplementary Health.

\section{Referências}

1. Brasil. Ministério da Saúde. Conselho Nacional de Secretários de Saúde. Sistema Único de Saúde. Brasília: Conass; 2011.

2. Conill EM. Sistemas comparados de saúde (2006) apud Campos GWS, Minayo MCS, Akerman M, Drumond JM, Carvalho YM. Tratado de saúde coletiva. Hucitec Fiocruz 2006; 880(1):563-613.

3. Mendes EV. Os grandes dilemas do SUS. Salvador: Casa da Qualidade; 2001.

4. Brasil. Lei no 9.961, de 28 de janeiro de 2000. Cria a Agência Nacional de Saúde Suplementar - ANS e dá outras providências. Diário Oficial da União, Brasília, 28 jan. 2000.

5. Brasil. Agência Nacional de Saúde Suplementar. Caderno de Informação da Saúde Suplementar: beneficiários, operadoras e planos. Rio de Janeiro: ANS; 2014

6. Brasil. Ministério da Saúde. Agência Nacional de Saúde Suplementar. Duas faces da mesma moeda: microrregulação e modelos assistenciais na saúde suplementar. Rio de Janeiro: ANS/MS; 2005.

7. Brasil. Agência Nacional de Saúde Suplementar. Planos odontológicos: evolução, desafios e perspectivas para a regulação da saúde suplementar. Rio de Janeiro: ANS; 2005.

8. Mussi DS. Saúde suplementar em odontologia: o caso de uma operadora de autogestão na cidade de Florianópolis [Dissertação de Mestrado]. Florianópolis: Universidade Federal de Santa Catarina; 2010.

9. Neumann DG. Modelo de atenção em saúde bucal na saúde suplementar: uma análise a partir das operadoras de planos odontológicos no Brasil [Tese de Doutorado]. Florianópolis: Universidade Federal de Santa Catarina; 2014.

10. Pietrobon L. Planos de saúde: uma análise das relações entre as operadoras, prestadores de serviço e beneficiários sob a visão do cirurgião-dentista [Tese de Doutorado]. Florianópolis: Universidade Federal de Santa Catarina; 2010.

11. Moraes FCB. Análise das relações entre prestadores de serviço e operadoras de planos odontológicos: visão do cirurgião-dentista [Trabalho de Conclusão de Curso]. Florianópolis: Universidade Federal de Santa Catarina; 2014.

12. Soares SCM. Avaliação da saúde bucal na Atenção Básica: a perspectiva do usuário [Dissertação de Mestrado]. Natal: Universidade Federal do Rio Grande do Norte; 2007.

13. Machline C, Serson FM, Belluomini A. Uma pesquisa de satisfação dos clientes de planos de saúde. In: $16^{\circ}$ Simpósio de Administração da Produção, Logística e Operações Internacionais: 2013; São Paulo. Anais. 2013. p. 10.

14. Brasil. Agência Nacional de Saúde Suplementar. Índice de Desempenho das Operadoras 2014. 2014 [acesso $2014 \mathrm{dez}$. 07]. Disponível em URL: http://www.ans.gov.br/espaco-da-qualidade/programa-de-qualificacao-de-operadoras.

15. Brasil. Agência Nacional de Saúde Suplementar. ANS TABNET: Informações em Saúde Suplementar. 2014 [acesso 2014 dez. 07]. Disponível em URL: http://www.ans.gov.br/ anstabnet/cgi-bin/dh?dados/tabnet_br.def.

16. Brasil. Agência Nacional de Saúde Suplementar. Relatório da Pesquisa de Satisfação dos Beneficiários. 2013 [acesso 2014 dez. 07]. Disponível em URL: http://www.ans.gov.br/ images/stories/Plano_de_saude_e_Operadoras/informacoes_avaliacoes/psb/psb_relatorio_completo_pesquisa.pdf.

17. Dawes J. Do data characteristics change according to the number of scale points used? An experiment using 5-point, 7-point and 10-point scales. Int J Mark Res 2008; 50(1):61-77.

18. Pinheiro RS, Torres TZG. Uso de servicos odontológicos entre os Estados do Brasil. Cien Saúde Colet 2006; 4(11):9991010. 
19. Barros AJD, Bertoldi AD. Desigualdades na utilização e no acesso a serviços odontológicos: uma avaliação em nível nacional. Cien Saúde Colet 2002; 7(4):709-17.

20. Gerschman S, Veiga L, Guimarães C, Ugá MAD, Portela MC, Vasconcellos MM, et al. Estudo de satisfação dos beneficiários de planos de saúde de hospitais filantrópicos. Cien Saúde Colet 2007; 12(2):487-500.

21. Cartaxo JLS, Santos MLR. Estudo da satisfação do usuário de planos de saúde odontológicos em Salvador no ano de 2004. Rev Baiana Saúde Pública 2007; 31(1):25-37.

22. Bahia L. Planos privados de saúde: luzes e sombras no debate setorial dos anos 90. Cien Saúde Colet 2001; 6(1):329-39.

23. Unidas Autogestão em Saúde. Pesquisa Nacional 2014. 2014 [acesso 2015 jan. 27]. Disponível em URL: http://www.unidas. org.br/uploads/WEB_pesquisa_unidas_21x28_141124.pdf.

24. Dutra RS. A arquitetura organizacional aplicada à construção de entidade de autogestão em saúde [Dissertação de Mestrado]. Porto Alegre: Universidade Federal do Rio Grande do Sul; 2006.

\section{Endereço para correspondência:}

Alyne Silveira Pereira

Rua Aureliano Coutinho, 259, apart. 1

Vila Buarque

01224-020 São Paulo, SP

Telefone: (11) 952199181

E-mail: alyneslvr@gmail.com

Recebido: 23/11/15. Aceito: 03/06/16. 